\title{
Comment: A Fruitful Resolution to Simpson's Paradox via Multiresolution Inference
}

\section{Citation}

Liu, Keli, and Xiao-Li Meng. 2014. “Comment: A Fruitful Resolution to Simpson's Paradox via Multiresolution Inference." The American Statistician 68 (1) (January 2): 17-29. doi:10.1080/00031305.2014.876842.

\section{Published Version}

doi:10.1080/00031305.2014.876842

\section{Permanent link}

http://nrs.harvard.edu/urn-3:HUL.InstRepos:17947657

\section{Terms of Use}

This article was downloaded from Harvard University's DASH repository, and is made available under the terms and conditions applicable to Open Access Policy Articles, as set forth at http:// nrs.harvard.edu/urn-3:HUL.InstRepos:dash.current.terms-of-use\#OAP

\section{Share Your Story}

The Harvard community has made this article openly available.

Please share how this access benefits you. Submit a story.

\section{Accessibility}




\title{
A Fruitful Resolution to Simpson's Paradox via Multi-Resolution Inference
}

\author{
Keli Liu and Xiao-Li Meng
}

\begin{abstract}
Simpson's Paradox is really a Simple Paradox if one at all. Peeling away the paradox is as easy (or hard) as avoiding a comparison of apples and oranges, a concept requiring no mention of causality. We show how the commonly adopted notation has committed the gross-ery mistake of tagging unlike fruit with alike labels. Hence, the "fruitful" question to ask is not "Do we condition on the third variable," but rather "Are two fruits which appear similar, actually similar at their core?". We introduce the concept of intrinsic similarity to sort through this mess. The notion of "core" depends on how deep one looks - the multi-resolution inference framework provides a natural way to define intrinsic similarity at the resolution appropriate for the treatment. To harvest the fruits of this insight, we will need to estimate intrinsic similarity, which often results in an indirect conditioning on the "third variable." A ripening estimation theory shows that the standard treatment comparisons, unconditional or conditional on the third variable, are low hanging fruit but often rotten. We pose assumptions to pluck away higher resolution (more conditional) comparisons - the multi-resolution framework allows us to rigorously assess the price of these assumptions against the resulting yield. One such assessment gives us Simpson's Warning: less conditioning is most likely to lead to serious bias when Simpson's Paradox appears.
\end{abstract}

\section{The Source of Confusions and Debates}

\subsection{Comparing Apples and Oranges}

Imagine Ms. Broken going to Dr. Heal to be treated for heart disease. A new treatment was made available to Dr. Heal, who also learned from a clinical trial that it can substantially outperform a standard treatment used as its control. However, its effectiveness depends on a patient's cholesterol level, which can also be altered significantly by the treatment. Therefore, to determine the appropriate treatment for Ms. Broken, Dr. Heal needs to know how trial subjects with cholesterol level similar to Ms. Broken's (say about $240 \mathrm{mg} / \mathrm{dL}$ ) responded to the two treatments. The clinical trial data did include cholesterol measurements for patients. Dr. Heal therefore seems to face a rather simple task: compare the treatment effects and side effects among those in the clinical trial with cholesterol level at $240 \mathrm{mg} / \mathrm{dL}$. Or using the terminology of Dr. Armistead's stimulating paper, Dr. Heal needs to condition on the third variable, cholesterol level, $Z=240$, when comparing the outcome variable $Y$ (e.g., $Y=1$ indicates success and $Y=0$ otherwise) between the treatment group (indicated by $T=1$ ) and the control group $(T=0)$.

So why all the fuss about whether or not to condition on the third variable $Z$ ? Since conditioning always leads to a more refined state space, shouldn't we always condition, at least in theory? The answer is yes, provided that we condition on the right conditions. In the scenario above, we were deliberately 
vague about "cholesterol measurements of patients," with the measurement time unspecified. Clearly to be relevant for Ms. Broken's choice, Dr. Heal should condition on cholesterol measurements taken at or just prior to the treatment. But what if the available data are post-treatment measurements? Suppose the new treatment decreases cholesterol by $20 \mathrm{mg} / \mathrm{dL}$, whereas the standard treatment has little impact. Then $Z_{\text {post }}=240$ corresponds to $Z_{a t}=260$ for the $T=1$ group, clearly incomparable with $Z_{a t}=Z_{\text {post }}=240$ for the $T=0$ group (assuming the treatment period is short enough that temporal effects are negligible). Therefore, conditioning on the same value of $Z_{\text {post }}$ actually leads to a comparison of apples and oranges: individuals alike post-treatment may be highly unlike at-treatment. This illustrates the rationale for Pearl's emphasis to not condition on variables affected by treatment.

However, the logical implication of "do not condition on variables affected by treatment" is not "condition on nothing". In our example, clearly we should condition on $Z_{a t}$, which, though unmeasured, could be deduced from $Z_{\text {post }}$ if we knew how the treatments acted on cholesterol. Thus, even if we should not condition on $Z_{\text {post }}$ itself, we should condition on a function of it. Third variables affected by treatments are therefore not useless; it is just that an extra processing step is required.

In practice, we typically do not have full knowledge about how $Z_{a t}$ impacts $Z_{\text {post }}$. However, as we shall demonstrate, even weak information or assumptions can lead to substantively higher quality comparisons than not conditioning (or improper conditioning). Our emphasis therefore is not to decide whether or when we should condition or not. Rather, we focus on the following more productive questions, and explore how the framework of multi-resolution inference (Meng, 2014) can help to answer them.

I. Ideal Question: If we had all the data we wished, what is the ideal (infinite resolution) conditioning that allows us to answer the substantive question exactly?

II. Inferential Question: How can we best approximate the ideal conditioning by an operational (finite resolution) conditioning (which may still not be directly estimable from the data)?

III. Estimation Question: How can we best estimate our operational conditioning based on the data we observe (data resolution)?

\subsection{In Case Causality is not Your Cup of Fruit ...}

Before we proceed, we echo Dr. Armistead's sentiment that the central issues of Simpson's paradox can be addressed adequately without necessarily invoking causality, unless one takes an encompassing view that no inference is complete without stating its causal origins or consequences. Causality is a useful tool for answering question (I), helping to define and identify the ideal "at-treatment" characteristics $Z_{a t}$ (Pearl, 2000). But it is not the only tool (see Section 2), and its overhead (e.g., familiarity with causal diagrams) may mask for some the fundamental motivation behind using it in the first place: avoid comparing apples and oranges. Besides, it provides little help for answering (II) or (III) which ask for a meaningful way to reduce the ideal set of $Z_{a t}$ to meet practical constraints. The easy answers, "include all $Z_{a t}$ " or "include all estimable $Z_{a t}$ ", turn out to be inadequate, as we shall demonstrate.

A moment's reflection on questions (I)-(III) shows that the inferential question is really a question about striking a balance between what we want to know (I) and what we can answer (III). Suppose you are standing at the edge of a lava pit with a treasure on an island in the pit's center. Pillars of obsidian jut out from the lava, and you can only jump on one before vaulting onto the island. But which one? Too close to the center may lead to a fiery death on your first jump; too far, and the same fate awaits on your second jump. This is precisely the "deathtrap" we face in choosing an operational conditioning. Yet mention of this tradeoff is absent from the usual discussions on Simpson's Paradox. Why is this?

The controversy surrounding "the third variable" has raged because we often focus on one of two 
questions to the exclusion of the other. The first asks, "What variables can we include in the operational conditioning?", the second "What operational conditionings can we estimate from the data?". Those who ask only the first question would conclude that causality solves everything. Others who worry only about the second would wonder why causality matters at all. But it is the third question, "What variables should we include in the operational conditioning?" that is most important and that conjoins the first two. Therefore, in the spirit of Dr. Armistead's paper, let us work towards a rebirth. But instead of the third variable, let us resurrect the "third question"! The multi-resolution setup in separating the ideal, the inferential, and the estimation questions directly addresses this forgotten question of "should".

\section{A Resolution Via Multiple Resolution}

Our motivating example shows that we can and should use $Z$ to infer the at-treatment similarity between subjects. While intuitive, the notion of at-treatment is clunky in that it suggests our reasoning depends on a temporal structure. In addition, all individuals are fundamentally unique, so what does similar mean? A formalism comprising the potential outcomes framework (see Rubin 2005) and the multi-resolution framework (see Meng, 2014) turns out to be adequate for formulating the meaning of at-treatment similarity. Wasserman (June 20, 2013, Blog) argued that the potential outcomes framework (or some causal variant) is necessary for understanding Simpson's Paradox. The gist of the matter is that we need to distinguish between the logical statements we wish to make (which are in terms of potential outcomes), and the probabilistic statements which we can estimate (which are in terms of observed data). This parallels our caveat above to distinguish the estimand $Z_{a t}$ from estimators based on $Z$. However, we would be complacent to simply warn against conditioning on $Z$ directly. We need to know how to condition on $Z$ indirectly but correctly. The multi-resolution framework addresses this deficiency.

\subsection{The Ideal Question: Infinite Resolution for Individuality}

Let $\Omega$ be our population of interest. Each $\omega \in \Omega$ represents an individual. All the intrinsic characteristics of this individual (e.g., age, sex, genomic signature, etc., when $\omega$ represents a human) are encoded into $\omega$. The idea behind the potential outcome framework is to imagine copies of these individuals in parallel universes where they receive different treatments. Similar to the setup in Pearl (2011), this can be formalized mathematically by considering an augmented product space $\Omega^{A} \equiv \Omega \times \mathbb{T}$, where $\mathbb{T}$ is the space of treatment assignments. A common setting is $\mathbb{T}=\{0,1\}$, as in our motivating example. We can never study any individual $\omega$ in isolation: our data are always produced from the individual in some universe. As a helpful analogy, one might think of $\omega$ as the Platonic form of the realized state ( $\omega, t)$ (of course, we cannot access the Platonic form directly). Anything we can observe are functions of the realized state, that is, $f(\omega, t)$, which could happen to be free of $t$ but such are special cases. The distinction between $\omega$ and $(\omega, t)$ is fundamental to what follows - as our invocation of Plato might suggest, what we really care about are properties of $\omega$, the form, and not properties of $(\omega, t)$, the realization of the form in a particular universe. To make this distinction clear, we will refer to $\omega$ as the individual and to $(\omega, t)$ as the state or realized state.

It is essential to understand that the assumption of the product space implies a decoupling between individuality, $\omega$, and the treatment. Hence intrinsic characteristics encoded in $\omega$ must remain invariant to treatment. The white-black plants example in Dr. Armistead's paper is useful for illustrating this point. Is color a treatment for the individual plant - is $\Omega$ in this case the population of plants? The product space assumption, $\Omega \times \mathbb{C}$ (where $\mathbb{C}$ is $\{$ white, black\}), says that we can choose any plant, $\omega$, and the state $(\omega, c)$ must be realizable in the world for all $c$. But color, $C(\omega)$, is purely a function of 
$\omega$, so the only realizable state is $(\omega, C(\omega))$. Hence the product space assumption, $\Omega \times \mathbb{C}$, is violated if $\Omega$ is the population of plants. Then what is $\Omega$ in this case? Suppose that there is a $1-1$ correspondence between the color of a plant and the first base pair in the plants genome, $g$ (a vector of base pairs). Suppose further that the support of possible $g$ is the product of the supports of $g$ 's components (this does not hold in practice but is used here for simplicity of illustration). Then we can decompose $g$ as $g=\left(c, g_{-1}\right)$ where $g_{-1}$ is $g$ with the first component removed. We can now let $\omega=g_{-1}$, so that $\Omega$ is the population of "proto"-plants. If we conceive of treatment as color, our unit of analysis is no longer plants but proto-plants. Any variable can be conceived of as a treatment, as long as we correctly identify the "individual" (and population) for which that variable is a treatment.

Having determined what our "individual" is, we can now ask whether we should condition on plant height when our treatment is color. As we discuss below, we should condition only on characteristics intrinsic to our individual - the appropriate conditioning depends on the unit of analysis. In this case, individual means proto-plant not plant. In the data, color is associated with plant height. Assuming that nature generated our sample of plants through independent sampling of proto-plants and colors, i.e., assuming a randomized experiment with proto-plants as units, our discussion below shows that this association implies that plant height functionally depends on color. Proto-plants lack color, hence, plant height cannot be an intrinsic characteristic of proto-plants. This is not to say that there isn't another characteristic called "proto-plant height" which is an intrinsic characteristic (and which we should condition on), but proto-plant height is different from the measured height (which is plant height). Conditioning on the latter does not lead to conditioning on the former.

In general, we do not know how nature creates plants from proto-plants and colors - that is to say when we conceive of the treatment as color we lack information on whether or not the observed data can be analyzed as a randomized experiment with proto-plants as the unit (or whether it should be seen as an observational study). Thus even though conceptually, comparing the effect of pesticides (with plants as the individual) is no different than comparing the effect of color (with proto-plants as the individual), having changed the unit of analysis, we lose (or gain) information on the generation of the data. Hence, comparing the effect of color is practically more challenging, because nature and not the scientist designs the experiment. This explains why we are fundamentally uncomfortable with seeing color as a "treatment" despite the fact that any variable is a treatment for some definition of "individual." It is not a treatment that we can apply.

To further emphasize the role played by the unit of analysis, note that each proto-plant, $\omega$, defines an equivalence class in the population of plants (one containing all plants with identical genome except possibly in the first base pair). So our unit of analysis is an individual when the population consists of proto-plants and an equivalence class when the population comprises plants. Clearly, proto-plant is a lower resolution unit of analysis than a plant. Hence, we can say that a treatment applied to individual plants (e.g., spraying pesticide) is of higher resolution than a treatment applied to equivalence classes of plants or proto-plants (e.g., color). The key to understanding what follows is that the correct conditioning should match the resolution of the treatment. In fact, another way to say "don't condition on variables affected by treatment" is "don't condition on characteristics which exceed the resolution of the treatment". That is, plant height, a characteristic of individual plants, exceeds the resolution of the treatment (color) which is applied to an equivalence class of plants. The multi-resolution perspective presents this crucial insight in its most transparent form: know your unit of analysis.

Whereas our setup is generic, for concreteness of discussion, we will focus on human populations and let $Y(\omega, t) \in\{0,1\}$ be the health status (e.g., cured or uncured) of state $(\omega, t)$. When an individual, $\omega^{*}$, walks into a doctor's office, ideally the doctor makes a choice of treatment by comparing $Y\left(\omega^{*}, 0\right)$ with 
$Y\left(\omega^{*}, 1\right)$, which obviously are unavailable. However this lack of direct data does not and should not deter us from formulating the question as the doctor asks it. Only after formulating the correct ideal question can we formulate the relevant inferential question. Throughout, we will also assume that the data are generated from a randomized experiment: the states $(\omega, t)$ which comprise our data are sampled through independent sampling of $\omega$ and $t$. Mathematically, this means that the treatment $T$ will be functionally independent of any function of $\omega$ alone, $f(\omega)$, a critical assumption for the discussions below.

\subsection{The Inferential Question: Finite Resolution for Similarity}

Our fundamental inference challenge - as Lindley and Novick (1981) stated - is to make a valid statement about $\left(Y\left(\omega^{*}, 0\right), Y\left(\omega^{*}, 1\right)\right)$ when we only observe $Y(\omega, 0)$ and $Y\left(\omega^{\prime}, 1\right)$ for some $\omega, \omega^{\prime} \neq \omega^{*}$. To address this problem, Lindley and Novick (1981) rely on the concept of exchangeability. We prefer the notion of resolution. The change in terminology suggests a different emphasis in action. Exchangeability is something we assume; resolution is something we can adjust. When we have no direct data (exact replications) to learn $Y\left(\omega^{*}, 0\right)$ or $Y\left(\omega^{*}, 1\right)$, we say that the resolution of the estimand, $\left(Y\left(\omega^{*}, 0\right), Y\left(\omega^{*}, 1\right)\right)$, exceeds the resolution of our dataset. In such cases, which include all clinical trials, we have to create approximate clones for $\omega^{*}$. The observed states of these approximate clones then provide indirect data (approximate replications) with which to infer $Y\left(\omega^{*}, 0\right)$ and $Y\left(\omega^{*}, 1\right)$. The resolution of our inference can be thought of as how strict we are in letting some $\omega$ be an approximate clone of $\omega^{*}$. The inferential question is: how strict should we be?

In finding an optimal strictness, we need to account for the error of approximating $\omega^{*}$ by $\omega$. How much do $\omega$ and $\omega^{*}$ differ with respect to intrinsic characteristics - those depending only on $\omega$ and $\omega^{*}$, and not their particular states, $(\omega, t)$ and $\left(\omega^{*}, t^{*}\right)$ ? As any good scientist would do, we wish to compare states with different treatments but intrinsically similar individuals. We are simply formalizing the scientific idea of ceteris paribus, holding all else $(\omega)$ constant except the treatment assignment. For $C(\omega, t)$, a realized-state characteristic, to also be an intrinsic characteristic, we must have $C(\omega, 0)=C(\omega, 1)$. Thus we say that a non-constant (vector) function $C$, defined on $\Omega^{A}=\Omega \times \mathbb{T}$, records an intrinsic characteristic of $\omega$ if $C(\omega, t)$ can be written as $C(\omega)$, i.e., $C$ is functionally independent of the treatment.

Equipped with this definition, we say that $\omega$ is an approximate clone for $\omega^{*}$, if for a selected set of intrinsic characteristics, $C(\omega)=C\left(\omega^{*}\right)$. That is, we define the $\omega^{*}$-relevant subpopulation with respect to $C$ as $\Omega_{C}\left(\omega^{*}\right)=\left\{\omega: C(\omega)=C\left(\omega^{*}\right)\right\}$. The resolution level, $R$, can then be defined as a numerical index of how restrictive this subpopulation is. A convenient choice is the dimension of $C$, with infinite resolution corresponding to cases where the only acceptable clone of $\omega^{*}$ is itself; see Meng (2014). Though flawed in many regards (e.g., it does not distinquish different kinds of infinite resolutions), this intuitive notion of resolution will suffice for our discussion of Simpson's Paradox. Our whole point is that it is wrong to define $\Omega_{C}\left(\omega^{*}\right)$ using a realized-state characteristic $C(\omega, t)$ that functionally depends on $t$ - this mistakes the fundamental unit of analysis as $(\omega, t)$ rather than $\omega$. As Plato would remind us, we should not care about superficial similarities $\left(C(w, t)=C\left(w^{\prime}, t^{\prime}\right)\right)$ but rather about intrinsic similarities $\left(C(w)=C\left(w^{\prime}\right)\right)$.

Example 1. Is it possible to define intrinsic similarity via the notion of independence most familiar to statisticians, i.e., stochastic independence? Intuitively, if " $T$ does not affect $Z$ ", then $Z$ is a property intrinsic to the individual rather than a consequence of treatment. This intuition is indeed correct, but equating "does not affect" with stochastic independence is not. To see this, let $Z_{\text {at }}$ be the standardized cholesterol level (in a population of interest) at the time of treatment such that $Z_{\text {at }} \sim N(0,1)$. Suppose the standardized post-treatment cholesterol level, $Z$, is linked to $Z_{\text {at }}$ via:

$$
Z=T\left(-Z_{\text {at }}\right)+(1-T)\left(Z_{\text {at }}\right)=(1-2 T) Z_{\text {at }} .
$$


Because $Z \mid T \sim N(0,1), Z$ is properly standardized conditionally and unconditionally. Consequently $Z$ is stochastically independent of $T$. Yet when we condition on $Z=z$ in the treatment group $T=1$, we obtain the subpopulation where $Z_{\text {at }}=-z$. In the control group $T=0$, however, restricting $Z=z$ would lead to the subpopulation where $Z_{\text {at }}=z$, clearly a rather different subpopulation from the one for $T=1$.

So what does stochastic independence give us? The stochastic independence of $T$ and $Z$ guarantees that Simpson's Paradox does not occur (Wasserman, June 20, 2013, Blog). The signs of the two comparisons, conditioning on $Z$ or not, will then agree. But this agreement itself says little about the validity of these comparisons. Indeed we would be misled if we take the agreement as a confirmation of validity. Only by conditioning on characteristics functionally independent of treatment can we guarantee a comparison of apples to apples. The downside of requiring functional independence between $Z$ and $T$, however, is that it cannot be tested by data. This echoes Pearl's emphasis (see Pearl, 2000, p.180) that probability calculus is not rich enough for handling Simpson's Paradox. Fortunately, when we use $Z$ to infer intrinsic characteristics, rather than for direct conditioning, we can circumvent this problem.

To proceed, we first note that at the resolution level defined by intrinsic characteristics, $C, \omega^{*}$ is indistinguishable from any individual in $\Omega_{C}\left(\omega^{*}\right)$. We can then approximate the infinite resolution estimand $\left(Y\left(\omega^{*}, 0\right), Y\left(\omega^{*}, 1\right)\right)$ by averaging $(Y(\omega, 0), Y(\omega, 1))$ over $\Omega_{C}\left(\omega^{*}\right)$ to obtain the lower resolution, operational estimand $P\left(Y(\omega, t)=1 \mid \omega \in \Omega_{C}\left(\omega^{*}\right)\right)$ for $t=0,1$. The inferential question, "How strict should we be?" becomes "How should we choose $C$ "? To increase the quality of our approximate clones, we want $C$ to be as rich a set of intrinsic characteristics as possible. But the price is a loss in our capacity to estimate the operational estimand from the observed data. For one, we may not observe all the components of $C$, and even when we do, there may not be any observed individual that satisfies $\omega \in \Omega_{C}\left(\omega^{*}\right)$; see Meng (2014). We therefore want our operational estimand $P\left(Y(\omega, t)=1 \mid \omega \in \Omega_{C}\left(\omega^{*}\right)\right)$ to be as close as possible to our ideal estimand, $Y\left(\omega^{*}, t\right)$, and simultaneously to approximate our operational estimand sufficiently well by an estimator. To strike the right balance, we need to understand the data resolution.

\subsection{The Estimation Question: Data Resolution}

Once we rigorously define the concept of intrinsic characteristics, there is no ambiguity over what variables to condition on: condition on as many intrinsic characteristics as possible. The question then turns to, "What is possible?". This is ultimately a problem of inferring intrinsic characteristics from observed data. The observation process, which we now define, determines the highest possible resolution for our inference, i.e., maximally what we can say about intrinsic similarity from apparent similarity.

Suppose our clinical trial contains data produced by $n$ realized states $\left\{\left(\omega_{i}, t_{i}\right)\right\}_{i=1}^{n}$. Let $C\left(\omega_{i}, t_{i}\right)$ denote a realized state (not necessarily intrinsic) characteristic. However, the potential outcomes $C_{1}\left(\omega_{i}\right) \equiv$ $C\left(\omega_{i}, 1\right)$ and $C_{0}\left(\omega_{i}\right) \equiv C\left(\omega_{i}, 0\right)$, are intrinsic characteristics of individual $\omega_{i}$ because these functions themselves are unaffected by the treatment assignment $t_{i}$. What is affected is which of these potential outcomes we are allowed to observe. Then $C\left(\omega_{i}, t_{i}\right)$ can be thought as being generated via:

$$
C\left(\omega_{i}, t_{i}\right)=t_{i} C_{1}\left(\omega_{i}\right)+\left(1-t_{i}\right) C_{0}\left(\omega_{i}\right)
$$

Clearly (2) is applicable whether $C$ is the dependent variable $Y$ or the third variable $Z$, as seen in (1).

To connect back to the common missing-data setup, we can view $Z\left(\omega_{i}, t_{i}\right)$ as the observed data and $\left(Z_{0}\left(\omega_{i}\right), Z_{1}\left(\omega_{i}\right)\right)$ as the missing or augmented data. We want to condition on the intrinsic characteristics $\left(Z_{0}\left(\omega_{i}\right), Z_{1}\left(\omega_{i}\right)\right)$, but this requires us to infer/predict it from $Z\left(\omega_{i}, t_{i}\right)$. By expressing $Z(\omega, t)$ in terms of $\left(Z_{0}(\omega), Z_{1}(\omega)\right)$ via $[2]$, we see that conditioning on the third variable leads to the comparison

$$
P(Y=1 \mid T=1, Z=z)-P(Y=1 \mid T=0, Z=z),
$$


which is mathematically equivalent to

$$
P\left(Y=1 \mid T=1, Z_{1}=z\right)-P\left(Y=1 \mid T=0, Z_{0}=z\right) .
$$

Whereas $(3)$ gives us the illusion of holding everything else constant other than the treatment assignment, the explicit differential subscripts in the higher resolution expression (4) reveal that we are actually comparing apples and oranges unless $Z_{0}(\omega)=Z_{1}(\omega)$ for all $\omega \in \Omega$. If we all adopted the explicit notation in (4), we believe much of the current confusion could have been avoided.

Obviously intrinsic characteristics generating no heterogeneity in the observed data cannot possibly distinguish any individuals in our data. Therefore, since by $2 \omega_{i}$ can affect the generation of $Z$ only through $\left(Z_{0}\left(\omega_{i}\right), Z_{1}\left(\omega_{i}\right)\right)$, we know that the choice $C\left(\omega_{i}\right)=\left(Z_{0}\left(\omega_{i}\right), Z_{1}\left(\omega_{i}\right)\right), R=2$, is the upper limit for how rich we can make $C\left(\omega_{i}\right)$. Thus we can take $R$ to be 0,1 , or 2 , but which one is optimal?

\subsection{Resurrecting The Third Question: $R=0,1$, or 2?}

Angrist et al. (1996) proposed studying subpopulations defined by all possible pairs of $\left(Z_{0}, Z_{1}\right)$, termed principal strata by Frangakis and Rubin (2002). In clinical trials with non-compliance, compliance can be thought of as a side effect of the treatment assignment (see Jin and Rubin, 2008), and hence we should compare treatments conditional on compliance type, $\left(Z_{0}, Z_{1}\right)$. Pearl (2011) also sees value in using principal strata to classify individuals. What he criticizes is their use in defining the notions of "direct" and "indirect effect". But when our primary objective is making a treatment choice for $\omega^{*}$, the opinion seems to be unanimous that conditioning on principal strata gives us a better look (relative to no conditioning) at how differences in treatment differ across individuals. Have we resolved Simpson's Paradox - is the answer always to choose $R=2$ with $C=\left(Z_{0}, Z_{1}\right)$ ?

Expression (2) with $C=Z$ shows that unless additional assumptions are made, we may only infer $Z_{0 i}$ when $T_{i}=0$ and $Z_{1 i}$ when $T_{i}=1$. Therefore, the overall data resolution, $R_{\text {data }}$, is 0 : no intrinsic characteristic is observed for all subjects. Thus to reach $R=2$, we need additional assumptions, typically in the form of prior specifications. But as usual, bias resulting from prior misspecification may overwhelm the resolutional benefit gained from using $R=2$ rather than $R=0$ or 1 . At the other end of the spectrum, we might be tempted to force $R=R_{\text {data }}=0$ for ease of estimation, but this choice throws away valuable information because for each individual $\omega_{i}$, we do observe one (and only one) component of $\left(Z_{0}\left(\omega_{i}\right), Z_{1}\left(\omega_{i}\right)\right)$. From this we cannot say which individuals are alike at resolution $R=2$, but we can say which are unlike: $\omega_{i}$ is unlike $\omega_{j}$ if $Z_{0}\left(\omega_{j}\right) \neq 0$ when $Z_{0}\left(\omega_{i}\right)=0$. As we see in Section 3 to exploit this partial information we will need to take $R>0$ even if $R_{\text {data }}=0$.

Just as we must carefully balance bias and variance in selecting a model, so we must pivot between the ideal high-resolution estimand and feasible low resolution estimators in choosing an operational estimand to net a better treatment decision. There is no such thing as a correct or natural choice of $C$. Box's quote "All models are wrong but some are useful" now becomes "All choices of $C$ are wrong (except $C(\omega)=\omega)$ but some are useful".

\section{Let's Enjoy Some Forgotten or Forbidden Fruits}

When a treatment decision is needed for an individual $\omega^{*}$, a key quantity of interest is $\theta\left(\omega^{*}\right)=$ $\operatorname{sign}\left\{Y_{1}\left(\omega^{*}\right)-Y_{0}\left(\omega^{*}\right)\right\}$, which indicates if treatment $T=1$ is better than $(\theta=1)$, worse than $(\theta=-1)$, or the same as treatment $T=0(\theta=0)$. The science of inference then is to choose a suitable population average to infer this individual-specific estimand, i.e., to approximate the ideal (infinite-resolution) estimand $\theta\left(\omega^{*}\right)$ by the operational estimand (a lower-resolution average), $E\left[\theta(\omega) \mid \omega \in \Omega_{C}\left(\omega^{*}\right)\right]$, using the 
notation of Section 2.2. However, this lower resolution average is itself not directly available since we never observe $\left(Y_{1}(\omega), Y_{0}(\omega)\right)$ jointly. Nevertheless, for binary $\left(Y_{0}, Y_{1}\right)$,

$$
E[\theta(\omega) \mid S]=E\left(Y_{1} \mid S\right)-E\left(Y_{0} \mid S\right)
$$

holds for any $S$ for which both sides of (5) are defined. This allows us to define an operational estimand:

$$
E\left[\theta(\omega) \mid \Omega_{C}\left(\omega^{*}\right)\right]=E\left(Y_{1}(\omega) \mid \omega \in \Omega_{C}\left(\omega^{*}\right)\right)-E\left(Y_{0}(\omega) \mid \omega \in \Omega_{C}\left(\omega^{*}\right)\right) .
$$

To estimate (6), we can then choose an estimator of the form

$$
\tilde{\theta}\left(\tilde{C}_{0}, \tilde{C}_{1}\right) \equiv E\left(Y_{1}(\omega) \mid \omega \in \Omega_{\tilde{C}_{1}}\left(\omega^{*}\right)\right)-E\left(Y_{0}(\omega) \mid \omega \in \Omega_{\tilde{C}_{0}}\left(\omega^{*}\right)\right)
$$

where $\tilde{C}_{0}, \tilde{C}_{1}$ can comprise only of intrinsic characteristics which are directly identifiable from data. To simplify our discussions, we have assumed in (7) that our samples are large enough that we can replace any sample average by the corresponding population average. Whereas we must choose the same $C$ in defining our operational estimand to ensure a comparison of apples and apples, we are unaware of any estimation principle that would prevent us from using different $\tilde{C}_{0}$ and $\tilde{C}_{1}$ in $(7)$. That is, $\tilde{C}_{0}$ and $\tilde{C}_{1}$ must both be sub-vectors of $C$, but they do not need to coincide. As long as our goal is correct, we can and should be as Machiavellian as possible in reaching it.

However, some readers might be puzzled or even disturbed by the idea of allowing different $\tilde{C}_{0}$ and $\tilde{C}_{1}$. What egregious hypocrites we are, accusing others of comparing apples and oranges, while our own prescription seems to advocate comparing apples to apricots! Have we warned others away from this forbidden fruit only to gorge on it ourselves? Of course not. What is forbidden is to mistake apparent similarity for intrinsic similarity, not the use of apparent dis-similarity to (better) estimate intrinsic similarity. We saw in Section 2.3 that if we force $\tilde{C}_{0}=\tilde{C}_{1}$, then the maximal resolution of $\tilde{C}_{0}=\tilde{C}_{1}$ (without further assumptions) is $R_{\text {data }}=0$. However, when $\tilde{C}_{0}$ and $\tilde{C}_{1}$ can differ, we can achieve $\tilde{R}_{0}>0$, $\tilde{R}_{1}>0$, where $\tilde{R}_{t}$ is the resolution of $\tilde{C}_{t}$; for an estimator $\tilde{\theta}\left(\tilde{C}_{0}, \tilde{C}_{1}\right)$ with $\tilde{R}_{0} \neq \tilde{R}_{1}$, we will denote its resolution as $\frac{1}{2}\left(\tilde{R}_{0}+\tilde{R}_{1}\right)$, leaving the question of the best designation to further research. This intuitive reasoning makes it easy to grasp why such an estimator can be better (e.g., having smaller MSE) than the one forcing $\tilde{C}_{0}=\tilde{C}_{1}$.

In Section 4.2 we will provide a theoretical condition, the " $1 / 2$ Rule" 26, justifying the use of $\tilde{C}_{0} \neq \tilde{C}_{1}$. The reality is that $\tilde{C}_{1-t}$ is missing from group $T=t$, so we cannot use it in predicting $Y_{t}$ (hence causing a mismatch in (7)). But can we pretend that we omitted $\tilde{C}_{1-t}$ not because it wasn't available but because it was not helpful (hence rendering the mismatch irrelevant)? The 1/2 Rule tells us when this pretense passes: the predictive power of what is missing must be less than half the predictive power of what is observed. The criterion used by the $1 / 2$ Rule is MSE. Hence it permits trading bias (incurred from our pretense) for variance (reduced by having $\tilde{R}_{0}, \tilde{R}_{1}>R_{\text {data }}$ ).

For concreteness, let $\tilde{C}_{t}=Z_{t}$, the post-treatment cholesterol under treatment $t$. If the success of treatment $t, Y_{t}$, depends mostly on how the patient's cholesterol changes with respect to that treatment and only somewhat on how the patient's cholesterol changes under the alternate treatment, then the $1 / 2$ Rule is satisfied. For those wondering why a patient's cholesterol under the alternate treatment might matter, such data can capture aspects of the patient's health status at time of treatment which affect the side effect of the alternate treatment but not the side effect of the treatment applied. The question then is to what extent those same aspects affect the main effect of the treatment applied. The 1/2 Rule aims to characterize when we can use the part of the data that is easy to use and ignore the part of the data that is "hard" to use-by hard we mean data whose use would require a full Bayesian model for $\left(Y_{0}, Y_{1}, Z_{0}, Z_{1}\right)$ thereby inviting (potentially very) biased prior information. 


\subsection{Low Resolution Estimand or Low Resolution Estimator?}

The conclusion that direct conditioning on the third variable, $(3)$, is valid only when $Z_{0}(\omega) \equiv Z_{1}(\omega)$ does not imply the next best alternative is no conditioning at all, that is, to use as the operational estimand:

$$
\theta_{R=0} \equiv P\left(Y_{1}=1\right)-P\left(Y_{0}=1\right)=P(Y=1 \mid T=1)-P(Y=1 \mid T=0),
$$

where the estimand resolution is $R=0$ because no intrinsic characteristics are used. Note (8) does not hold in general without assuming that $T$ is independent of $\left(Y_{0}, Y_{1}\right)$, as emphasized by Wasserman (June 20,2013 , Blog). As argued before, even if it is not legitimate to condition on $Z$, conditioning on the two-component (and hence $R=2$ ) intrinsic characteristic $\left(Z_{0}, Z_{1}\right)$ gives us a valid operational estimand:

$$
\theta_{R=2}\left(z_{0}, z_{1}\right) \equiv P\left(Y_{1}=1 \mid Z_{0}=z_{0}, Z_{1}=z_{1}\right)-P\left(Y_{0}=1 \mid Z_{0}=z_{0}, Z_{1}=z_{1}\right),
$$

which is a better approximation to our ideal estimand $\theta\left(\omega^{*}\right)$ than is 8 . The question now becomes whether we can find a good enough estimator of (9) to exploit its higher resolution or whether the higher cost of estimating $(9)$, as compared to $(8)$, represents too large an investment. Let $\tilde{R}$ denote the resolution of an estimator for $\theta_{R=2}$. From this perspective, we see that the assumption, $Z_{0}=Z_{1}$, is really the most convenient condition for achieving $\tilde{R}=2$ by reducing $[9$ to 3 , which permits the simplest estimation procedure. Simplicity is always welcome in practice, but must be assessed against the possible invalidity of too strong a condition. The multi-resolution framework reminds us that weaker conditions do exist, and that $Z_{0}=Z_{1}$ is not the only assumption that can motivate us to use (9) instead of (8).

To simplify our discussion, let us assume that $Z_{t}$ 's are binary, as in Dr. Armistead's paper (e.g., $Z=0$ and $Z=1$ indicate respectively whether patient's post-treatment blood pressure remained low or became normal). If individual $i$ is assigned to treatment, $T_{i}=1$, observing $Z_{i}=1$ allows us to infer $Z_{1 i}=1$, but not whether the individual belongs to subpopulation $\left\{\left(Z_{0}, Z_{1}\right)=(0,1)\right\}$ or to subpopulation $\left\{\left(Z_{0}, Z_{1}\right)=(1,1)\right\}$. Given this reality, we have two strategies:

i. Lower the resolution, $R$, of our operational estimand.

ii. Estimate our high resolution estimand, $(9)$, by using a lower resolution estimator, $\tilde{R}<R$.

The consequence of either choice is resolution bias - a mismatch of desired versus adopted resolution. For (i), the resolution bias is incurred in the decision phase (when we use the operational estimand to make a decision), whereas for (ii), it is incurred in the inference phase. Let $D_{\tilde{\theta}} \equiv D(\tilde{\theta})$ be a decision function taking estimates, $\tilde{\theta}$, of $\theta\left(\omega^{*}\right)=\operatorname{sign}\left\{Y_{1}\left(\omega^{*}\right)-Y_{0}\left(\omega^{*}\right)\right\}$ as argument and let $L\left(D_{\theta\left(\omega^{*}\right)}, D^{\prime}\right)$ be the loss of decision $D^{\prime}$ when the optimal decision is $D_{\theta\left(\omega^{*}\right)}$. Adopting the notation in the previous section, the two types of resolution bias are

$$
\begin{array}{cl}
\text { Inference Bias: } & \tilde{\theta}\left(\tilde{C}_{0}, \tilde{C}_{1}\right)-E\left[\theta(\omega) \mid \Omega_{C}\left(\omega^{*}\right)\right] \\
\text { Decision Bias: } & L\left(D_{\theta\left(\omega^{*}\right)}, D_{E\left[\theta(\omega) \mid \Omega_{C}\left(\omega^{*}\right)\right]}\right) .
\end{array}
$$

The bias we ultimately hope to minimize is

$$
\text { Realized Bias: } \quad L\left(D_{\theta\left(\omega^{*}\right)}, D_{\tilde{\theta}\left(\tilde{C}_{0}, \tilde{C}_{1}\right)}\right)
$$

One can think about minimizing 12 directly, of course, but this reduced formulation masks the pivotal role of $C$, the mechanism through which we actually can influence the realized bias. To conceptually connect the realized bias back to the more helpful I-bias and D-bias, we rewrite the realized bias as

$$
\begin{aligned}
L\left(D_{\theta\left(\omega^{*}\right)}, D_{\tilde{\theta}\left(\tilde{C}_{0}, \tilde{C}_{1}\right)}\right) & =L\left(D_{\theta\left(\omega^{*}\right)}, D_{E\left[\theta(\omega) \mid \Omega_{C}\left(\omega^{*}\right)\right]}\right) \\
& +\Delta_{L} \cdot \mathbb{I}\left\{\left|\tilde{\theta}\left(\tilde{C}_{0}, \tilde{C}_{1}\right)-E\left[\theta(\omega) \mid \Omega_{C}\left(\omega^{*}\right)\right]\right|>\tau\right\} \\
& =\text { D-bias }+ \text { Estimation Penalty } \cdot \mathbb{I}\{\text { I-bias }>\text { Tolerance }\}
\end{aligned}
$$


$\Delta_{L}$ is the change in loss if $D_{\tilde{\theta}\left(\tilde{C}_{0}, \tilde{C}_{1}\right)}$ were used instead of $D_{E\left[\theta(\omega) \mid \Omega_{C}\left(\omega^{*}\right)\right]}$ (the optimal decision being $\left.D_{\theta\left(\omega^{*}\right)}\right)$ and $\tau$ is the amount of I-bias needed for our actual decision to deviate from the intended decision (under the operational estimand), i.e., $D_{\tilde{\theta}\left(\tilde{C}_{0}, \tilde{C}_{1}\right)} \neq D_{E\left[\theta(\omega) \mid \Omega_{C}\left(\omega^{*}\right)\right]}$. Identity $[13$ is conceptually powerful because it reveals the balancing role played by $C$ which appears in both terms - the choice of $C$ must balance the error from using a non-ideal estimand for decision making (11) and the estimation error for that non-ideal estimand 10 .

This choice between (i) and (ii) is reminiscent of the bias-variance tradeoff. However, the bias-variance tradeoff takes place entirely within the inference phase, whereas the tradeoff between I-bias and D-bias occurs across phases. The utility of this two phase setup is to remind us that for coarse decisions, e.g., treatment 1 versus treatment 0, a large amount of I-bias can be incurred without changing our final decision. On the other hand, D-bias by definition alters our decision from the optimum. The tolerance term, $\tau$, captures this asymmetry in how I-bias and D-bias enter into the realized bias, hence distinguishing it from the usual bias-variance tradeoff. Expression 13 is most useful for binary decisions as it employs a single penalty and tolerance term. When the decision space is richer, (13) may be rewritten to exhibit additional thresholds (with associated penalties). But the emphasis is the same: we may prefer making stronger assumptions to estimate a higher resolution operational estimand using low resolution data - knowing full well that this estimate will be biased - over settling for a low resolution operational estimand. The latter, even though estimated with certainty, may yet be meaningless or misleading.

\subsection{Simpson's Warning and the ID-Bias Tradeoff}

We begin our investigation of the ID-bias tradeoff with strategy (ii). Whereas the success rate of treatment 1 is observed in the superpopulation $\left\{Z_{1}=z_{1}\right\}$, the corresponding rates in its two subpopulations, $\left\{\left(Z_{0}, Z_{1}\right)=\left(0, z_{1}\right)\right\}$ and $\left\{\left(Z_{0}, Z_{1}\right)=\left(1, z_{1}\right)\right\}$, though desired, are not. Under the usual mean-squared loss, the best prediction of the desired $P\left(Y_{1}=1 \mid Z_{0}, Z_{1}=z_{1}\right)$, as a function of the random variable $Z_{0}$, is its expectation conditional on $Z_{1}=z_{1}$, which is the superpopulation success rate $P\left(Y_{1}=1 \mid Z_{1}=z_{1}\right)=P\left(Y=1 \mid T=1, Z=z_{1}\right)$. This is equivalent to choosing $C=\left(Z_{0}, Z_{1}\right)$ in 6 and $\tilde{C}_{1}=Z_{1}$ in (7). Similarly for $T=0$, we choose $\tilde{C}_{0}=Z_{0}$; as discussed, to estimate conditioning on $\Omega_{C}\left(\omega^{*}\right)$ we can choose $\Omega_{\tilde{C}_{0}}\left(\omega^{*}\right) \neq \Omega_{\tilde{C}_{1}}\left(\omega^{*}\right)$.

Applying this reasoning, we can estimate the $R=2$ estimand 9 by the $\tilde{R}=1$ estimator

$$
\tilde{\theta}_{R=2}^{\tilde{R}=1}\left(z_{0}, z_{1}\right)=P\left(Y=1 \mid T=1, Z=z_{1}\right)-P\left(Y=1 \mid T=0, Z=z_{0}\right), \quad z_{0}, z_{1} \in\{0,1\} .
$$

The use of the tilde notation $\tilde{\theta}$ instead of the usual hat notation $\hat{\theta}$ is to remind us that even if there is no sampling error - 14 is a population mean instead of sample average - we will still have errors caused by the discrepancy between $\tilde{R}$ and $R$. Also note that an implicit assumption here is that both values of $\{0,1\}$ are observed for $Z$ under $T=1$ and $T=0$; in general we assume the support of $Z_{t}$ is invariant to $t$ (which may be violated, such as when the treatment shifts all cholesterol level upward).

Expression (14) says that in order to conclude that treatment 1 is superior to treatment 0 for all principal strata $\left\{\left(Z_{0}, Z_{1}\right)=\left(z_{0}, z_{1}\right)\right\}$, we need to consider four separate comparisons. For the two comparisons with $z_{0}=z_{1}$, our estimate $\tilde{\theta}_{R=2}^{\tilde{R}=1}\left(z_{0}, z_{1}\right)$ corresponds to exactly the two $Z$-conditional contrasts, (3). Thus the $Z$-conditional contrasts have forgotten about the two principal strata with $Z_{0} \neq Z_{1}$, where individuals observed to be dissimilar in the two treatment groups may be actually intrinsically similar (though we must be mindful of our I-bias in making this assertion). Of course, if all four comparisons share the same sign, then we pay no price for our forgetfulness (if we base our treatment decision only on the sign). This is precisely the situation where Simpson's Paradox does not occur. If all four comparisons implied by 14 are positive, we obtain $P(Y=1 \mid T=1)>P(Y=1 \mid T=0)$. Hence either the 
$Z$-conditional contrasts or the marginal contrast, taken as a reduction of (14), preserves the full sign information contained in [14.

When Simpson's Paradox occurs, the sign of the estimated treatment effect on the subpopulation where $Z_{0}=Z_{1}$ must differ from the sign of the estimated treatment effect over the subpopulation where $Z_{0} \neq Z_{1}$. The $Z$-conditional contrasts contain the sign information for the subpopulation where $Z_{0}=Z_{1}$, and the marginal contrast contains the sign information for the subpopulation where $Z_{0} \neq$ $Z_{1}$. Neither tells the entire story. Simpson's Paradox is not paradoxical at all from this viewpoint: the sign of the $Z$-conditional contrast, (3), and the sign of the marginal contrast, 8), do not contain contradictory information, but rather orthogonal information pertaining to disjoint subpopulations. Or as Dr. Armistead put it "an apparent contradiction that may contain more than one truth."

Ironically, the advice to use only the marginal contrast when we cannot assume $Z_{0}=Z_{1}$, makes the same mistake as the advice to use the $Z$-conditional contrast. They both throw away the sign information for parts of the population. Conceptually, we fell into the trap of thinking that either (8) or (3) must be correct, when they are both correct or incorrect, depending on which individuals the information will be applied to. The appearance of Simpson's Paradox provides evidence for treatment effect by subpopulation interaction. A low resolution estimand, e.g. (8), will incur high D-bias, because the optimal decision may differ across principal strata. Instead of a paradox, the lesson we are given is Simpson's Warning:

Low resolution operational estimands are most dangerous (higher D-bias) when Simpson's Paradox appears.

Rather than telling us to default to a marginal comparison when $Z_{0} \neq Z_{1}$, the appearance of Simpson's Paradox is a sign that we should consider taking on I-bias to make a high resolution inference, e.g. (14), accounting for treatment effect by subpopulation interactions. However, in any particular situation, one may still feel that the I-bias incurred from using $\tilde{\theta}_{R=2}^{\tilde{R}=1}$ to estimate $\theta_{R=2}$ trumps any reduction in D-bias.

To make a more satisfactory ID-bias tradeoff, we can choose an operational estimand with resolution between $\theta_{R=0}$ and $\theta_{R=2}$. We can form marginal principal strata defined by $Z_{0}$ and $Z_{1}$ individually instead of jointly. This leads to operational estimands at resolution $R=1$ :

$$
\theta_{R=1, Z_{t}}(z)=P\left(Y_{1}=1 \mid Z_{t}=z\right)-P\left(Y_{0}=1 \mid Z_{t}=z\right),
$$

for $t=0,1$. When our target is $\theta_{R=1, Z_{0}}$, because $Z_{0 i}$ is observed for everyone assigned to treatment 0 , we can estimate the $P\left(Y_{0}=1 \mid Z_{0}=z\right)$ term in 15 with no I-bias by setting $\tilde{C}_{0}=Z_{0}$. For individuals in the treatment 1 group, we observe $Z_{1 i}$ but not $Z_{0 i}$. In the absence of prior knowledge of the correlation between $Z_{0 i}$ and $Z_{1 i}$, we once again estimate subpopulation rates using superpopulation rates, that is, we set $\tilde{C}_{1}$ to be empty in $(7)$. The price of this strategy is I-bias but by lowering the resolution of our estimand to $R=1$, we need only pay this price for the $P\left(Y_{1}=1 \mid Z_{0}=z\right.$ ) term in 15 (though logically it is possible for the difference of two biased estimators to be unbiased for the difference of their estimands). Our estimator then is

$$
\tilde{\theta}_{R=1, Z_{0}}^{\tilde{R}=0.5}(z)=P(Y=1 \mid T=1)-P\left(Y=1 \mid T=0, Z_{0}=z\right),
$$

with resolution $\tilde{R}=\left(\tilde{R}_{0}+\tilde{R}_{1}\right) / 2=0.5$. In contrast to 14 , which requires four comparisons, 16 requires only two comparisons, reflecting the increased D-bias caused by lowering our resolution. Specifically, at resolution $R=1$, we take into account how the treatment effect may change across subpopulations defined by $Z_{0}(=0,1)$ but ignore further changes in the treatment effect within those subpopulations.

In a nutshell, the resolution framework urges us to carefully consider the risk of adopting subpopulation specific but I-biased estimators versus robust but D-biased estimators. In addition, the resolution framework reminds us that $\theta_{R=0}$ and $\theta_{R=2}$ are not the only possible ways to compare the treatments. 


\subsection{Increase Data Resolution via Eliminating Subpopulations}

In practice, some combination of lowering the estimand resolution and increasing the data resolution may be necessary to achieve a satisfactory ID-bias tradeoff. First, we note that the strong assumption $Z_{0}=Z_{1}$ increases the estimation resolution to $\tilde{R}=2$ by ruling out two subpopulations: $\left\{\left(Z_{0}, Z_{1}\right)=(0,1)\right\}$ and $\left\{\left(Z_{0}, Z_{1}\right)=(1,0)\right\}$. This assumption reduces the dimension of our estimand to make it identified. But we can weaken this assumption by ruling out only a single subpopulation, achieving estimators with resolution $\tilde{R}=1.5$. We will incur I-bias in estimating $\theta_{R=2}$ but gain some robustness. Specifically, we can make the following "no-defier" assumption (see Angrist et al., 1996).

Exclusion Assumption: The subpopulation defined by $\left\{\left(Z_{0}, Z_{1}\right)=(1,0)\right\}$ is empty.

In the context of blood pressure, this assumption says that treatment 1 performs at least as well as treatment 0 in raising the patient's blood pressure. A plausible scientific story is the existence of an unobserved genetic factor $G$, where $G=0,1,2$ represent respectively the homozygote recessive, the heterozygote and the homozygote dominant individuals. The homozygotes recessive and dominant are disposed towards low and normal blood pressure respectively, regardless of treatment. The heterozygote is not predisposed towards either low or normal blood pressure - in this case, blood pressure is decided by treatment rather than genetic causes. Specifically, we have

$$
Z=\left\{\begin{array}{ll}
0 & \text { if } G=0 \\
T & \text { if } G=1 \\
1 & \text { if } G=2
\end{array} \Rightarrow \begin{array}{l}
\left\{\left(Z_{0}, Z_{1}\right)=(0,0)\right\}=\{G=0\} \\
\left\{\left(Z_{0}, Z_{1}\right)=(0,1)\right\}=\{G=1\} \\
\left\{\left(Z_{0}, Z_{1}\right)=(1,1)\right\}=\{G=2\}
\end{array}\right.
$$

Consequently, conditioning on principal strata allows us to successfully condition on the appropriate genetic factor even though it is unobserved and possibly even unknown to us. The non-existence of the subpopulation $\left\{\left(Z_{0}, Z_{1}\right)=(1,0)\right\}$ is induced by the ternary nature of genotypes. Whereas this story is useful for explaining the intuition behind principal strata, our calculations below do not depend on it.

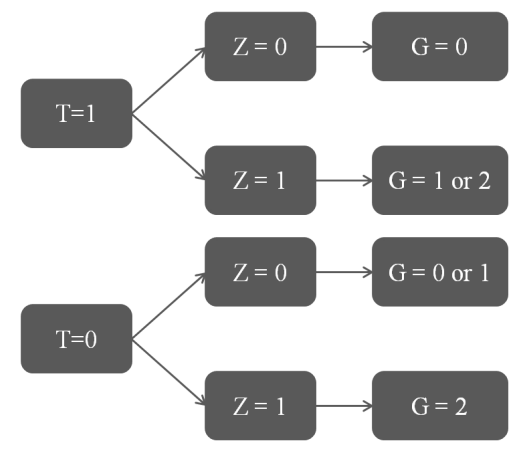

Figure 1: Inferring membership in principal strata from observed data.

As Figure 1 shows, by eliminating one principal stratum, we can directly infer $\left(Z_{0 i}, Z_{1 i}\right)$ from $T_{i}$ and $Z_{i}$ whenever $T_{i} \neq Z_{i}$. Hence the exclusion assumption increases the resolution of our data. But even for those individuals with $T_{i}=Z_{i}$, we still have some information on their likely stratum membership. To see this, denote $p_{i j}=P\left(Z_{0}=i, Z_{1}=j\right)$ for $i, j=0,1$. Then the exclusion assumption implies that

$$
p_{00}=P\left(Z_{1}=0\right)=P(Z=0 \mid T=1) \quad \text { and } \quad p_{11}=P\left(Z_{0}=1\right)=P(Z=1 \mid T=0) .
$$

This allows us to estimate $\left\{p_{i j}, i, j=0,1\right\}$ directly from the data, because $p_{10}=0$ and $p_{01}=1-p_{00}-p_{11}$. 
Next we see that under the exclusion assumption we need to make only three comparisons:

$$
\theta_{R=2}(g)=P\left(Y_{1}=1 \mid G=g\right)-P\left(Y_{0}=1 \mid G=g\right) \equiv \pi_{1 g}-\pi_{0 g}, \quad g=0,1,2 .
$$

To estimate $\left\{\pi_{t g}\right\}$ for $t=0,1$ and $g=0,1,2$, let $\mu_{t, z}=P(Y=1 \mid T=t, Z=z)$. We then have

$$
\begin{gathered}
\mu_{1,0}=\pi_{10}, \quad \mu_{0,1}=\pi_{02} ; \\
\mu_{0,0}=\frac{p_{00}}{p_{00}+p_{01}} \cdot \pi_{00}+\frac{p_{01}}{p_{00}+p_{01}} \cdot \pi_{01}, \quad \mu_{1,1}=\frac{p_{01}}{p_{01}+p_{11}} \cdot \pi_{11}+\frac{p_{11}}{p_{01}+p_{11}} \cdot \pi_{12} .
\end{gathered}
$$

Because all four $\mu_{t, z}$ 's are directly estimable from the observed data (assuming we have observations with both values of $Z$ under either treatment), $\pi_{10}$ and $\pi_{02}$ are in turn directly estimable because of (19), but $\pi_{00}, \pi_{01}, \pi_{11}$ and $\pi_{12}$ are not because the equations in 20 are under-determined. Thus our estimator will have resolution $\tilde{R}<2$ and incur I-bias in estimating $\theta_{R=2}$.

However, for making a treatment decision, we may need only to infer $\operatorname{sign}\left(\theta_{R=2}\right)$. Therefore, even if we do not know each $\left(\pi_{0 g}, \pi_{1 g}\right)$ exactly, we may still able to determine whether or not $\pi_{1 g}>\pi_{0 g}$, eliminating I-bias for estimating $\operatorname{sign}\left(\theta_{R=2}\right)$. For example, let $o_{1 \mid 01}=p_{01} / p_{00}$ (odds of $G=1$ given $G=0$ or 1 ) and $o_{1 \mid 12}=p_{01} / p_{11}$ (odds of $G=1$ given $G=1$ or 2 ) yield respectively

$$
\pi_{00}=\left(1+o_{1 \mid 01}\right) \mu_{0,0}-o_{1 \mid 01} \pi_{01}, \quad \pi_{12}=\left(1+o_{1 \mid 12}\right) \mu_{1,1}-o_{1 \mid 12} \pi_{11} .
$$

Using the fact that all $\pi_{t g}$ 's must stay inside $[0,1]$, the first equation above restricts $\pi_{00}$ to the interval

$$
\max \left\{0, \mu_{0,0}\left(1+o_{1 \mid 01}\right)-o_{1 \mid 01}\right\} \leq \pi_{00} \leq \min \left\{1,\left(1+o_{1 \mid 01}\right) \mu_{0,0}\right\}
$$

and similarly we can derive bounds for $\pi_{12}$. Such bounds, if sufficiently tight, allow for estimation of $\operatorname{sign}\left(\theta_{R=2}\right)$ even when $\theta_{R=2}$ is not directly estimable. Furthermore, even if the bounds do not lead to a definite estimate of $\operatorname{sign}\left(\theta_{R=2}\right)$, they may still enable us to make essentially bias-free decisions at a higher resolution level with the help of extremely weak prior information, as we demonstrate below.

\begin{tabular}{cccc}
$Z=0$ & $Y=0$ & $Y=1$ & $n$ \\
$($ Low BP $)$ & & & \\
\hline$T=0$ & 21 & 9 & 30 \\
$T=1$ & 8 & 2 & 10 \\
\hline Totals & 29 & 11 & 40
\end{tabular}

\begin{tabular}{cccc}
$Z=1$ & $Y=0$ & $Y=1$ & $n$ \\
$($ Normal BP) & & & \\
\hline$T=0$ & 3 & 7 & 10 \\
$T=1$ & 12 & 18 & 30 \\
\hline Totals & 15 & 25 & 40
\end{tabular}

Table 1: Lindley-Novick Dataset

Example 2. Table 1 gives the Lindley-Novick dataset, as modified in Dr. Armistead's paper. For simplicity, we will treat it as the population of interest instead of merely a sample and hence we can ignore any hat notation (but retain the tilde notation as needed). As noted in the paper, as a side effect, treatment 1 appears to raise blood pressures more than treatment 0 does, so the exclusion assumption is not contradicted by the data. Adopting this assumption, by 17 we obtain that $p_{00}=p_{11}=10 / 40=0.25$ and hence $p_{01}=0.5$. That is, half the population comprises of individuals for whom treatment 1 was more effective than treatment 0 in increasing the blood pressure and the other half have blood pressure invariant to treatment choice. Equations 19 and 20 now become

$$
\begin{aligned}
0.2=\mu_{1,0}=\pi_{10}, & 0.7 & =\mu_{0,1}=\pi_{02} ; \\
0.3=\mu_{0,0}=\frac{1}{3} \pi_{00}+\frac{2}{3} \cdot \pi_{01}, & 0.6 & =\mu_{1,1}=\frac{2}{3} \pi_{11}+\frac{1}{3} \pi_{12} .
\end{aligned}
$$


Using 23, we can derive bounds for the remaining success rates:

$$
0 \leq \pi_{00} \leq 0.9, \quad 0 \leq \pi_{01} \leq 0.45, \quad 0.4 \leq \pi_{11} \leq 0.9, \quad 0 \leq \pi_{12} \leq 1
$$

These bounds are loose. Hence we are unable to directly conclude from them a definite $\operatorname{sign}$ for $\theta_{R=2}(g)=$ $\pi_{1 g}-\pi_{0 g}$. (Section 4.1 will provide a direct link of this phenomenon to Simpson's Paradox.)

The closest we come to a definitive conclusion is in the subpopulation $\left\{\left(Z_{0}, Z_{1}\right)=(0,1)\right\}$, where we can conclude that $\theta_{R=2}(1) \geq-.05$, which suggests rather strong evidence that it is more likely than not that $\theta_{R=2}(1) \geq 0$. As a matter of fact, from [23), we see that $\theta_{R=2}(1)<0$ if and only if $\pi_{12}-\pi_{00}>0.9$, which requires $\pi_{12}>90 \%$ and $\pi_{00}<10 \%$. However, if this were a real life application, doctors can usually ballpark the magnitude of the success rate for various treatments. What is unknown is a more fine-scale comparison between alternatives. Yet this weak prior information (e.g., common sense) alone may be sufficient to rule out extreme rates such as $\pi_{12}>90 \%$ or $\pi_{00}<10 \%$ as well as their opposite nature. Putting all the pieces together, this analysis says that we cannot be sure which treatment is better for those whose blood pressure will be equally affected by both treatments. However, there is rather strong evidence that the main advantage of treatment 1 over treatment 0 , if it exists, is through the superior effect of treatment 1 in raising blood pressure, echoing Dr. Armistead's finding.

\subsection{What Fruits Are Worth the I-Bias Price?}

When bounds of the form 22 are insufficient for estimating $\operatorname{sign}\left(\theta_{R=2}\right)$ and no useful prior information is available, we can resort, as before, to estimating subpopulation rates using their superpopulation counterparts. For the $T=0$ group, we can use the superpopulation $\left\{Z_{0}=0\right\}$ to cover the estimations for $\left\{Z_{0}=0, Z_{1}=0\right\}$ and $\left\{Z_{0}=0, Z_{1}=1\right\}$, that is, we set $\tilde{C}_{0}=Z_{0}$ in 7 when estimating $\pi_{00}$ and $\pi_{01}$. Similarly, we set $\tilde{C}_{1}=Z_{1}$ when estimating $\pi_{11}$ and $\pi_{12}$. For cases where a subpopulation is directly observed, we set $\tilde{C}_{0}=\tilde{C}_{1}=\left(Z_{0}, Z_{1}\right)$. As in 14 , the price of this estimation is I-bias.

Example 2 (continued). Adopting the strategy above, we obtain

$$
0.3=\mu_{0,0}=\tilde{\pi}_{00}=\tilde{\pi}_{01}, \quad 0.6=\mu_{1,1}=\tilde{\pi}_{11}=\tilde{\pi}_{12}
$$

We can then use them to construct estimates for $\theta_{R=2}$ (note $\pi_{10}$ and $\pi_{02}$ are directly available at resolution 2 under the exclusion assumption and hence they do not "wear" tilde):

$$
\tilde{\theta}_{R=2}^{\tilde{R}=1.5}(0,0)=\pi_{10}-\tilde{\pi}_{00}=-0.1, \quad \tilde{\theta}_{R=2}^{\tilde{R}=1}(0,1)=\tilde{\pi}_{11}-\tilde{\pi}_{01}=0.3, \quad \tilde{\theta}_{R=2}^{\tilde{R}=1.5}(1,1)=\tilde{\pi}_{12}-\pi_{02}=-0.1 .
$$

Here the estimator resolution for the principal stratum $\left\{Z_{0}=0, Z_{1}=0\right\}$ is $\tilde{R}=1.5$ since $\tilde{C}_{1}=C=$ $\left(Z_{0}, Z_{1}\right)\left(\tilde{R}_{1}=2\right)$ and $\tilde{C}_{0}=Z_{0}\left(\tilde{R}_{0}=1\right)$. A similar logic gives the estimator resolution for the principal stratum $\left\{Z_{0}=1, Z_{1}=1\right\}$. The stratum $\left\{Z_{0}=0, Z_{1}=1\right\}$ has a lower estimator resolution, $\tilde{R}=1$, because both terms must be estimated: $\tilde{C}_{0}=Z_{0}\left(\tilde{R}_{0}=1\right)$ and $\tilde{C}_{1}=Z_{1}\left(\tilde{R}_{1}=1\right)$.

Our estimates reiterate that treatment 1 outperforms treatment 0 only when it does a better job in raising blood pressure. In fact, for individuals whose blood pressure is invariant to $T$, statistically speaking, treatment 1 actually fares worse. Under our exclusion assumption, $50 \%$ of individuals have blood pressure that is invariant to treatment choice ${ }^{1}$ A marginal comparison hides this information. While the low resolution operational estimand, $\theta_{R=0}=(20 / 40)-(16 / 40)=0.1$, is robust, it is misleading because it ignores treatment effect by subpopulation interactions. By characterizing these interactions,

\footnotetext{
${ }^{1}$ This does not mean that treatment 1 is the wrong choice for $50 \%$ of new patients - a conclusion that relies on the fallacy of the electoral college: winning a majority in subpopulations comprising a majority of the superpopulation does not imply winning a majority of the superpopulation.
} 
we see that the doctor should try hard to ascertain whether the patient's blood pressure will be invariant to treatment choice, and make a decision accordingly. For example, if the genotype $G$ truly regulates blood pressure according to our story, a doctor could ascertain $\left(Z_{0}\left(\omega^{*}\right), Z_{1}\left(\omega^{*}\right)\right)$ prior to making a treatment decision through genetic screening (but this requires us to at least suspect the genetic effect, perhaps through a secondary study).

But what if we truly have no predictive accuracy for the value of $\left(Z_{0}\left(\omega^{*}\right), Z_{1}\left(\omega^{*}\right)\right)$ ? Let us consider a case where $\theta_{R=0}>0$ but we know nothing about the value of $Z_{0}\left(\omega^{*}\right)$ or $Z_{1}\left(\omega^{*}\right)$ except that they are equal (e.g., we cannot predict a patient's blood pressure after either treatment, but we have good reasons to believe that the impact of the treatment on the blood pressure, as a side effect, will be very similar). If we must select a treatment, the best decision - in terms of minimizing the probability of mistake-is to assign $\omega^{*}$ to treatment 1 , which is wholly based on $\theta_{R=0}$. However, $\theta_{R=2}$ is not useless - the higher resolution information tells us about the quality and risk of the decision based on $\theta_{R=0}$. In particular, if $\theta_{R=0}(0,0)>0$ and $\theta_{R=0}(1,1)>0$, then we know our decision based on $\theta_{R=0}$ is reliable in that it is invariant to any new information about $\left(Z_{0}, Z_{1}\right)$. But if $\theta_{R=0}(0,0)$ and $\theta_{R=1}(1,1)$ are of opposite sign, then our decision will not be invariant to information on $\left(Z_{0}, Z_{1}\right)$ - the lack of invariance measures the inadequacy of low resolution information. This same logic generalizes to the case where $Z_{0} \neq Z_{1}$. In our example, a decision based on $\theta_{R=0}$ will be invariant to new information on $\left(Z_{0}, Z_{1}\right)$ only $50 \%$ of the time, i.e., it is no more reliable than flipping a fair coin.

When the decision process is no longer binary but includes the option "gather more information," information on the risk of choosing treatment 1 (or 0 ) can be used directly in decision making. Since intrinsic characteristics are functionally independent of treatment, conceptually nothing prevents us from assessing $\left(Z_{0}, Z_{1}\right)$ prior to treatment; after all, the treatment process itself is a particular measurement process which uses $T$ to tease out $\left(Z_{0}, Z_{1}\right)$ - a process that always creates missing data depending on $T$. We can, for example, use a patient's medical history or genetic screening to predict $\left(Z_{0}\left(\omega^{*}\right), Z_{1}\left(\omega^{*}\right)\right)$. That is, an estimate of $\theta_{R=2}$ can lead to a different decision even when $\left(Z_{0}\left(\omega^{*}\right), Z_{1}\left(\omega^{*}\right)\right)$ is unknown, if the former makes us suspect that a treatment decision based on $\theta_{R=0}$ alone is of unacceptable quality.

Example 2 (continued). We explicitly quantify how the additional information in our estimate of $\theta_{R=2}$ translates into tighter bounds for the error probability of a decision based on $\theta_{R=0}$. For any subpopulation $S$, define $q_{i j}^{S}=P\left(Y_{0}=i, Y_{1}=j \mid \omega \in S\right)$ for $i, j=0,1$. Let $q_{.1}^{S}=q_{01}^{S}+q_{11}^{S}$ and $q_{1}^{S}=$ $q_{10}^{S}+q_{11}^{S}$. Define the error rate of choosing treatment 1 for subpopulation $S$ as

$$
\varepsilon^{S}(1) \equiv q_{10}^{S} /\left(q_{10}^{S}+q_{01}^{S}\right)
$$

This gives the probability that we will make the incorrect decision when our choice leads to different outcomes, i.e., when it matters. Suppose that we wish to bound $\varepsilon^{S}$ (1) given $\theta^{S} \equiv q_{01}^{S}-q_{10}^{S}$, for a subpopulation, $S$; note $\theta^{S}$ is directly observable from data because

$$
P\left(Y_{0}=0, Y_{1}=1 \mid \omega \in S\right)-P\left(Y_{0}=1, Y_{1}=0 \mid \omega \in S\right)=P\left(Y_{1}=1 \mid \omega \in S\right)-P\left(Y_{0}=1 \mid \omega \in S\right) .
$$

Then we find the maximum and minimum (over $\left.q_{10}^{S}\right)$ of $\varepsilon^{S}(1)=q_{10}^{S} /\left(2 q_{10}^{S}+\theta^{S}\right)$. For the Lindley-Novick data, we want the error rate over the entire population since nothing is known about our patient. We first estimate this rate using only information at resolution $R=0$. We observe $\theta=\theta_{R=0}=0.1$. In addition, $0 \leq q_{10} \leq q_{1}$., where $q_{1}$. can be estimated by $P\left(Y_{0}=1\right)=0.4$. This leads to bounds $0 \leq \varepsilon(1) \leq 4 / 9$.

Moving to higher resolution (but estimated with I-bias) information at resolution $R=2$, we see that since $\tilde{\theta}_{R=2}^{\tilde{R}=1.5}(0,0)=-0.1, \sqrt{25}$ implies that $0.1 \leq q_{10}^{(0,0)}$, where $S$ here is the subpopulation $\left\{Z_{0}=0, Z_{1}=0\right\}$. Similarly $\tilde{\theta}_{R=2}^{\tilde{R}=1.5}(1,1)=-0.1$ implies $0.1 \leq q_{10}^{(1,1)}$. The lower bound for $q_{10}^{(0,1)}$ cannot be improved from 0 since $\tilde{\theta}_{R=2}^{\tilde{R}=1.5}(0,1)=0.3$. Using these bounds together with the fact $p_{00}=p_{11}=0.25$ 
and $p_{01}=0.5$ yields an improved lower bound for $q_{10}=\sum_{i, j} q_{10}^{(i, j)} p_{i j} \geq 0.05$. We then minimize and maximize $\varepsilon(1)=q_{10} /\left(2 q_{10}+\theta\right)$ over $0.05 \leq q_{10} \leq 0.4$ for $\theta=0.1$ to obtain $1 / 4 \leq \varepsilon(1) \leq 4 / 9$.

By incorporating higher resolution information we substantially improve the lower bound for $\varepsilon(1)$-in particular, the bounds using only low resolution information are too optimistic about treatment 1 . In the absence of information about $\left(Z_{0}\left(\omega^{*}\right), Z_{1}\left(\omega^{*}\right)\right)$ for our new patient, our best decision (when we cannot "gather more information") is still to choose treatment 1 , i.e., we still base our decision on $\theta_{R=0}$. But the higher resolution information allows us to ascertain the uncertainty of our decision. A fitting analogy to classical statistics is the difference between a point estimate and a confidence interval. (It is doubly fitting because the uncertainty ascertainment itself is subject to error: it relies on lower resolution estimators such as $\tilde{\theta}_{R=2}^{\tilde{R}=1.5}$, just as we typically estimate the variance term when constructing a confidence interval.)

\subsection{A Compromising Resolution Without Compromising Inference}

When we use I-biased estimates, as in the previous section, we obviously should worry about the sensitivity of our inferences to the I-bias incurred. One way to ascertain this sensitivity is to lower the resolution of our operational estimand-hence lowering the I-bias. We can do so by focusing on $\theta_{R=1, Z_{0}}$ rather than on $\theta_{R=2}$. The marginal stratum $\left\{Z_{0}=0\right\}$ is a mix of the principal strata $\left\{\left(Z_{0}, Z_{1}\right)=(0,0)\right\}$ and $\left\{\left(Z_{0}, Z_{1}\right)=(0,1)\right\}$, equivalently $\{G=0\}$ and $\{G=1\}$, whereas $\left\{Z_{0}=1\right\}=\{G=2\}$. We have lowered the I-bias because the first equation in 20 becomes $\mu_{0,0}=P\left(Y_{0}=1 \mid Z_{0}=0\right)$, permitting a direct estimation of the control success rate in $\left\{Z_{0}=0\right\}$. We no longer need bounds for $\pi_{00}$ and $\pi_{01}$, effectively reducing the undetermined parameters to just $\pi_{11}$ and $\pi_{12}$ in 20 . This reduction is especially powerful if $\pi_{00}$ or $\pi_{01}$ were the quantities which we could not sufficiently bound. If it was $\pi_{11}$ or $\pi_{12}$ in 20 for which practical bounds did not exist, then we should consider using $\theta_{R=1, Z_{1}}$ instead of $\theta_{R=1, Z_{0}}$. If neither equation in 20 provides useful bounds, then non-negligible I-bias accrues even at resolution $R=1$.

Example 2 (continued). Let $\varphi_{t z}=P\left(Y_{t}=1 \mid Z_{0}=z\right)$. Then

$$
\varphi_{10}=\frac{1}{3} \pi_{10}+\frac{2}{3} \pi_{11}, \quad \varphi_{00}=0.3, \quad \varphi_{11}=\pi_{12}, \quad \varphi_{01}=0.7 .
$$

Our inability to bound $\pi_{12}$ in (24) means that $\varphi_{11}$ remains unidentified. Thus we cannot compare the two treatments for the marginal stratum $\left\{Z_{0}=1\right\}$ without incurring I-bias. However, since $\pi_{10}=\mu_{1,0}=$ 0.2 and $0.4 \leq \pi_{11} \leq 0.9$ (from (24)), by substituting this information into the equation for $\varphi_{10}$, we obtain $1 / 3 \leq \varphi_{10} \leq 2 / 3$. This implies that $\varphi_{00}=0.3<\varphi_{10}$. Thus, statistically speaking, treatment 1 outperforms treatment 0 if a patient's blood pressure remains low under treatment 0 . This conclusion is reached without I-bias and relies only on the exclusion assumption. In addition, we know that the population proportion of such individuals is $P\left(Z_{0}=0\right)=0.75$. This allows us to say that even if $Z_{0}$ were known for our patient (and even if we somehow discovered the value of $\varphi_{11}$ ), there is at least a $75 \%$ chance that we would not change our decision from the one based on $\theta_{R=0} L^{2}$

Therefore, as before, the higher resolution information allows us to calibrate the reliability of a decision based on $\theta_{R=0}$ - reliability in the sense of invariance of our decision to new sources of knowledge. This assessment of reliability comes without I-bias, but it does come with D-bias in that we can only speak about decision invariance to newfound information about $Z_{0}$, not the more refined $\left(Z_{0}, Z_{1}\right)$. By lowering our resolution, we ignore that the treatment effect may differ in the subpopulations $\left\{Z_{0}=0, Z_{1}=0\right\}$ and $\left\{Z_{0}=0, Z_{1}=1\right\}$. The decrease in I-bias at the cost of D-bias explains why our conclusion here differs from our conclusion in Section 3.4 that there is only a $50 \%$ chance that our decision will be invariant to

\footnotetext{
${ }^{2}$ Again, this differs from the incorrect assertion that treatment 1 will outperform treatment 0 with probability at least $75 \%$.
} 
new knowledge. However, both inferences carry much more (and higher quality) information than if we had chosen $\theta_{R=0}$ as our operational estimand, i.e., if we had simply compared $P(Y=1 \mid T=1)=0.5$ to $P(Y=1 \mid T=0)=0.4$ and concluded that treatment 1 is better on average. Information on the quality (and risk) of our decision carried in the higher resolution inference but missing from the lower resolution operational estimand can be quite valuable when designing long term treatment plans in practice.

\section{More Fruit for Thoughts}

\subsection{A Warning and Also a Dilemma: Scylla or Charybdis?}

The loose bounds for $\left(\pi_{00}, \pi_{01}, \pi_{11}, \pi_{12}\right)$ in the Lindley-Novick dataset indicate that the data are ambiguous about the comparative treatment effectiveness for specific principal strata $\left\{\left(Z_{0}, Z_{1}\right)=\left(z_{0}, z_{1}\right)\right\}$. The slackness of these bounds turns out to be directly related to the presence of Simpson's Paradox. In fact, we have the following result:

Suppose that Simpson's Paradox occurs in a dataset. Then we will not be able to bound $\left(\pi_{00}, \pi_{01}, \pi_{11}, \pi_{12}\right)$ to guarantee $\pi_{1 g} \geq \pi_{0 g}$ for $g=0,1,2$, even under the exclusion assumption.

To prove this, first note that by the law of total probability, $\pi_{1 g} \geq \pi_{0 g}$ for $g=0,1,2$ implies $P\left(Y_{1}=1\right) \geq P\left(Y_{0}=1\right)$ and hence $P(Y=1 \mid T=1) \geq P(Y=1 \mid T=0)$ for a randomized experiment. We now show that this contains enough information for the following statement to also hold for $z=0,1$ :

$$
\mu_{1, z} \equiv P(Y=1 \mid T=1, Z=z) \geq P(Y=1 \mid T=0, Z=z) \equiv \mu_{0, z}
$$

For the bound $\pi_{00} \leq \pi_{10}$ to hold for all values of $\pi_{01}$, by the first expression in $(21)$, we must have

$$
\max _{\pi_{01}} \pi_{00}=\mu_{0,0}\left(1+o_{1 \mid 01}\right) \leq \pi_{10}=\mu_{1,0}
$$

which implies $\mu_{1,0} \geq \mu_{0,0}$. Similarly for the bound $\pi_{12} \geq \pi_{02}$ to hold for all values of $\pi_{11}$, by the second expression in 21, we require

$$
\min _{\pi_{11}} \pi_{12}=\mu_{1,1}\left(1+o_{1 \mid 12}\right)-o_{1 \mid 12} \geq \pi_{02}=\mu_{0,1}
$$

Rewriting this inequality gives

$$
\mu_{1,1} \geq \mu_{0,1}+\frac{o_{1 \mid 12}}{1+o_{1 \mid 12}}\left(1-\mu_{0,1}\right) \geq \mu_{0,1}
$$

Hence the marginal and $Z$-conditional contrasts share a common sign.

The result says that if Simpson's paradox occurs, then the data, even with our exclusion assumption, does not contain sufficient evidence for the superiority of treatment 1 over treatment 0 across all nonempty subpopulations. This statement has two sides. First, we see again that Simpson's Paradox occurs precisely when the better treatment may differ across subpopulations. The presence of this interaction reduces the utility of low resolution operational estimands such as $\theta_{R=0}$, which give us no information about the quality/risk of any decision we make. The D-bias of low resolution estimands will be high. Is there a way to make a high resolution inference in this case without incurring significant I-bias?

The second side of the statement answers this question in the negative. We cannot hope to make a decision at resolution $R=2$ relying on only the bounds supplied by the exclusion assumption. We will either have to take on I-bias by using superpopulation averages to estimate subpopulation averages or lower the resolution of our operational estimand, say to $R=1$. Thus, there is a genuine tradeoff 
between I-bias and D-bias to be made. At this juncture, we must make the decision whether to sail closer to Scylla or Charybdis. For the Lindely-Novick data, we were able to reach a healthy compromise by comparing two treatments within subpopulations defined by $Z_{0}$ alone (a resolution $R=1$ inference). We found statistical evidence for treatment 1 to be superior for individuals with low blood pressure under treatment 0 . This conclusion is free of I-bias and with less D-bias than the operational estimand $\theta_{R=0}$.

Simpson's Warning foreshadows not just the potential for D-bias but dashes any hopes for reducing D-bias without incurring some I-bias. We need to strike a resolution-robustness compromise to obtain the most useful and reliable decision. We feel that the current thinking leans too heavily in favor of minimizing I-bias: defaulting to the resolution zero estimand, $P\left(Y_{1}=1\right)-P\left(Y_{0}=1\right)$. This is a bad habit of statisticians in thinking only in terms of estimation error - we would rather estimate a bad model correctly than estimate a good one poorly. Certainly we should take some advice from crafty Odysseus who opted to lose a few men to Scylla rather than his whole crew to Charybdis. In our minds, the D-bias of low resolution estimands is Charybdis - how could we make the right decision if we are asking the wrong questions? The I-bias from trying to obtain a higher resolution inference is the few men we must sacrifice to Scylla to save the decision making enterprise as a whole.

\subsection{The Nightshade Is Actually A Tomato}

In Section 3 we proposed estimators for $\theta=Y_{1}-Y_{0}$ of the form (7), which maximize the resolution component-wise. That is, in treatment group $T=t$, we approximate the population $\Omega_{C}\left(\omega^{*}\right)$ by $\Omega_{\tilde{C}_{t}}\left(\omega^{*}\right)=\Omega_{C_{t}}\left(\omega^{*}\right)$ where $C_{t}$ contains all intrinsic characteristics observed for that group. The alternative chooses identical approximating populations for both groups: $\Omega_{\tilde{C}_{0}}\left(\omega^{*}\right)=\Omega_{\tilde{C}_{1}}\left(\omega^{*}\right)=\Omega_{C_{\text {com }}}\left(\omega^{*}\right)$, conditioning only on those intrinsic characteristics observable in both treatment groups, leading to the estimator

$$
\tilde{\theta}\left(C_{\mathrm{com}}\right) \equiv E\left[Y_{1}(\omega) \mid \omega \in \Omega_{C_{\mathrm{com}}}\left(\omega^{*}\right)\right]-E\left[Y_{0}(\omega) \mid \omega \in \Omega_{C_{\mathrm{com}}}\left(\omega^{*}\right)\right]=E\left[\theta \mid C_{\mathrm{com}}\right]
$$

Note that $\Omega_{C_{0}}\left(\omega^{*}\right)$ and $\Omega_{C_{1}}\left(\omega^{*}\right)$ are both refinements of $\Omega_{C_{\text {com }}}\left(\omega^{*}\right)$. The possible superiority of the maximal component-wise resolution estimator, $\tilde{\theta}\left(C_{0}, C_{1}\right)$, over $\tilde{\theta}\left(C_{\mathrm{com}}\right)$ underpins the justification for choosing an operational estimand with resolution greater than the data resolution. Hence, the theory of such estimators will be a crucial component in the future development of multi-resolution inference. Below, we offer some low-hanging but nonetheless rich fruit to hopefully entice others.

Our goal is to characterize when $\tilde{\theta}\left(C_{0}, C_{1}\right)$ dominates $\tilde{\theta}\left(C_{\mathrm{com}}\right)$ in MSE. In evaluating the frequentist properties of $\tilde{\theta}\left(C_{0}, C_{1}\right)$ and $\tilde{\theta}\left(C_{\text {com }}\right)$ it makes sense to condition on $C_{\text {com. }}$. To simplify notation, let $\hat{Y}_{t} \equiv E\left[Y_{t}(\omega) \mid C_{t}\right], R_{t} \equiv Y_{t}-\hat{Y}_{t}, \sigma_{t}^{2} \equiv V\left(\hat{Y}_{t} \mid C_{\text {com }}\right)$, and for $t=0,1$,

$$
\beta_{t \mid 1-t}^{\mathrm{obs}} \equiv \frac{\operatorname{Cov}\left(\hat{Y}_{t}, \hat{Y}_{1-t} \mid C_{\mathrm{com}}\right)}{V\left(\hat{Y}_{1-t} \mid C_{\mathrm{com}}\right)} \equiv \frac{\sigma_{01}^{\mathrm{obs}}}{\sigma_{1-t}^{2}}, \quad \beta_{t \mid 1-t}^{\mathrm{mis}} \equiv \frac{\operatorname{Cov}\left(R_{t}, \hat{Y}_{1-t} \mid C_{\mathrm{com}}\right)}{V\left(\hat{Y}_{1-t} \mid C_{\mathrm{com}}\right)} \equiv \frac{\sigma_{t, 1-t}^{\mathrm{mis}}}{\sigma_{1-t}^{2}} .
$$

Then $\tilde{\theta}\left(C_{0}, C_{1}\right)$ attains smaller MSE than $\tilde{\theta}\left(C_{\mathrm{com}}\right)$ if and only if $\sigma_{1,0}^{\mathrm{mis}}+\sigma_{0,1}^{\mathrm{mis}} \leq \frac{1}{2}\left(\sigma_{0}^{2}+\sigma_{1}^{2}\right)-\sigma_{01}^{\mathrm{obs}}$. To help us interpret, consider the case $\sigma_{0}^{2}>0, \sigma_{1}^{2}>0$ which allows us to rewrite the condition in terms of the $1 / 2$ Rule:

$$
\frac{\sigma_{0}^{2}}{\sigma_{0}^{2}+\sigma_{1}^{2}} \beta_{1 \mid 0}^{\mathrm{mis}}+\frac{\sigma_{1}^{2}}{\sigma_{0}^{2}+\sigma_{1}^{2}} \beta_{0 \mid 1}^{\mathrm{mis}} \leq \frac{1}{2}\left[\frac{\sigma_{0}^{2}}{\sigma_{0}^{2}+\sigma_{1}^{2}}\left(1-\beta_{1 \mid 0}^{\mathrm{obs}}\right)+\frac{\sigma_{1}^{2}}{\sigma_{0}^{2}+\sigma_{1}^{2}}\left(1-\beta_{0 \mid 1}^{\mathrm{obs}}\right)\right] .
$$

$\sigma_{t}^{2}$ is the amount of variation in $Y_{t}$ explained by the observed data $C_{t}$. So the weight $\sigma_{t}^{2} /\left(\sigma_{0}^{2}+\sigma_{1}^{2}\right)$ is the fraction of total explained variation attributable to $C_{t}$. But how much of this information is unique to 
$C_{t}$, i.e., would we have done any worse if the situation had been reversed and we predicted $Y_{t}$ using $C_{1-t}$ instead of $C_{t}$ ? To answer, we regress this $\hat{Y}_{t}$ on a function of $C_{1-t}, \hat{Y}_{1-t}$. If $\hat{Y}_{1-t}$ is perfectly redundant for the information in $C_{t}$, then the regression coefficient $\beta_{t \mid 1-t}^{\text {obs }}$ equals 1 . So $1-\beta_{t \mid 1-t}^{\text {obs }}$ measures the predictive power for $Y_{t}$ unique to the observed data, $C_{t}$. Hence, the right hand side of 26] equals $1 / 2$ times a weighted average of the predictive information unique to the observed data for $Y_{0}$ and $Y_{1}$ respectively. Similarly, we can calculate the predictive power for $Y_{t}$ that is unique to the missing data, $C_{1-t}$. Note that $R_{t}$ is variation in $Y_{t}$ unexplained by $C_{t}$. Hence the regression coefficient of $R_{t}$ on $\hat{Y}_{1-t}$ measures how much information in $C_{1-t}$ is missing from $C_{t}$. The left-hand side of 26 is a weighted average of the predictive information unique to the missing data. Thus condition 26 can be interpreted as

$$
\text { Info Unique to Missing Data } \leq \frac{1}{2} \text {. Info Unique to Observed Data. }
$$

Consider the special case where $\Omega_{C_{0}}\left(\omega^{*}\right)=\Omega_{C_{\text {com }}}\left(\omega^{*}\right)$, i.e., the resolutional improvement is concentrated in predicting $Y_{1}$. Then, we require $\beta_{0 \mid 1}^{\mathrm{mis}} \leq \frac{1}{2}$, i.e., the information we neglected to use in the group $T=0$ should not be highly predictive of the residuals $Y_{0}-E\left(Y_{0} \mid C_{\mathrm{com}}\right)$.

The danger of choosing $\tilde{C}_{0} \neq \tilde{C}_{1}$ in 26 is that the missing data may be highly influentialinferences which condition only on the observed data will then fail to adequately approximate the operational estimand which conditions on both the observed and missing data. However, if dependence of outcome on the missing data is weak - in fact less than $1 / 2$ the dependence of outcome on the observed data - then we can essentially "ignore the error term" and still enjoy the resolutional benefits of choosing our operational estimand so that $R=\operatorname{dim}(C)>\operatorname{dim}\left(C_{\text {com }}\right)$. To make this interpretation clearer, we illustrate 26) on a canonical example.

Example 3. For analytic tractability, both $\left(C_{0 i}, C_{1 i}\right)$ and $\left(Y_{0 i}, Y_{1 i}\right)$ are taken to be continuous, though the intuition flows back easily to the discrete case. Assume that $\left(C_{0 i}, C_{1 i}\right)$ are standardized but correlated normal variates: $N_{2}\left(\mathbf{0}_{2},(1-\rho) \mathbf{I}_{2}+\rho \mathbf{1}_{2} \mathbf{1}_{2}^{T}\right)$, which affect the potential outcomes $\left(Y_{0 i}, Y_{1 i}\right)$ via

$$
Y_{t}=\mu_{t}+\alpha^{\mathrm{obs}} C_{t}+\alpha^{\mathrm{mis}} C_{1-t}+\varepsilon_{t}
$$

for $t=0,1$, where $\varepsilon_{0}$ and $\varepsilon_{1}$ are iid $N\left(0, \tau^{2}\right)$ and independent of $\left(C_{0 i}, C_{1 i}\right)$. In the treatment group we observe only $C_{1}$ and in the control group we observe only $C_{0}$. Thus $C_{\text {com }}$ is empty. The question of interest: when is $E\left(Y_{1} \mid C_{1}\right)-E\left(Y_{0} \mid C_{0}\right)$ a better predictor of $Y_{1}-Y_{0}$ (in MSE terms) than $E\left(Y_{1}\right)-E\left(Y_{0}\right)$ ? As suggested, the answer depends on how important the missing information, $C_{1-t}$, is compared to the observed information, $C_{t}$, in predicting $Y_{t}$, determined here by the values of $\left(\alpha^{\text {obs }}, \alpha^{\text {mis }}\right)$.

The best (in MSE terms) predictor of $Y_{t}$ using $C_{t}$ and the prediction residual are

$$
\hat{Y}_{t}=E\left(Y_{t} \mid C_{t}\right)=\mu_{t}+\left(\alpha^{\mathrm{obs}}+\alpha^{\mathrm{mis}} \rho\right) C_{t} ; \quad R_{t}=Y_{t}-\hat{Y}_{t}=\alpha^{\mathrm{mis}}\left(C_{1-t}-\rho C_{t}\right)+\varepsilon_{t}
$$

To write condition 26 , we find

$$
1-\beta_{t \mid 1-t}^{\mathrm{obs}}=\frac{1-\rho^{2}}{1+\rho} \quad \beta_{t \mid 1-t}^{\mathrm{mis}}=\frac{1-\rho^{2}}{\alpha^{\mathrm{obs}} / \alpha^{\mathrm{mis}}+\rho} .
$$

These regression coefficients measure the predictive information unique to the observed and missing data respectively - the similarity in form is striking with $\alpha^{\text {obs }} / \alpha^{\text {mis }}$ replacing the constant 1 in the latter. When $\alpha^{\text {obs }}>0, \alpha^{\text {mis }}>0$, then $\beta_{t \mid 1-t}^{\text {mis }}$ is a monotonic decreasing function of $\alpha^{\text {obs }} / \alpha^{\text {mis }}$ - the ratio of the predictive strength of the observed data to that of the unobserved data. Since $\sigma_{0}=\sigma_{1}$, 26, then has the form

$$
\frac{1-\rho^{2}}{\alpha^{\text {obs }} / \alpha^{\text {mis }}+\rho} \leq \frac{1}{2} \frac{1-\rho^{2}}{1+\rho} .
$$

When $\rho \geq 0$, this becomes $\alpha^{\text {obs }} / \alpha^{\text {mis }} \geq 2+\rho$. So if $\rho=0$, we need the information in the observed data $C_{t}$ to be twice as important as the information in the missing data, $C_{1-t}$. In many applications this is not an 
unreasonable assumption. After all, we would expect the potential side effects experienced by our patient under treatment $t$ to be much more predictive of the success/failure of treatment $t$ than the potential side effects experienced under alternative treatments. In these situations maximizing component-wise resolution will lead to smaller MSE than adopting the data resolution, namely, using the unconditional comparison $E\left(Y_{1}\right)-E\left(Y_{0}\right)$ to predict $Y_{1}-Y_{0}$.

The limitation of 26 is that it applies only when MSE adequately describes our actual loss. For real life examples, other loss functions may be more appropriate: in selecting treatment for a patient, our focus may be 0-1 loss (did we choose the right or wrong treatment?). Nevertheless, the 1/2 Rule establishes the possibility of improving our decision making by maximizing resolution component-wise. And while the magic number may differ from $1 / 2$ for other losses, the intuition remains: the explanatory power of the observed data must trump that of the missing data. We hope this insight will encourage others to taste this previously forbidden fruit, which is more tomato (yes, a tomato is a fruit) than nightshade. We ought to stop now before we over serve our dessert after Dr. Armistead's main entree,

which was rich with food for thought. We do, however, hope that our multi-resolution fruit basket is large and inviting enough for readers who remain hungry for more - please help yourself!

\section{Acknowledgements}

We thank Dr. Armistead for an invigorating article which stimulated us to take a higher resolution look at Simpson's Paradox. Jessica Hwang provided invaluable advice on organization and structure, as well as pointing out incoherencies and inconsistencies. Of course, the remaining incoherencies and inconsistencies are entirely our own. We also thank the NSF for partial financial support, and the editor, Ronald Christensen, and journal manager, Eric Sampson, for their saintly patience amid growing despair.

\section{References}

Joshua D Angrist, Guido W Imbens, and Donald B Rubin. Identification of causal effects using instrumental variables. Journal of the American Statistical Association, 91(434):444-455, 1996.

Constantine E Frangakis and Donald B Rubin. Principal stratification in causal inference. Biometrics, $58(1): 21-29,2002$.

Hui Jin and Donald B Rubin. Principal stratification for causal inference with extended partial compliance. Journal of the American Statistical Association, 103(481):101-111, 2008.

Dennis V Lindley and Melvin R Novick. The role of exchangeability in inference. The Annals of Statistics, 9(1):45-58, 1981.

Xiao-Li Meng. A trio of inference problems that could win you a Nobel Prize in statistics (if you help fund it). In Xihong Lin, David L. Banks, Christian Genest, Geert Molenberghs, David W. Scott, and Jane-Ling Wang, editors, In The Past, Present and Future of Statistical Science, pages 535-560. CRC Press, 2014.

Judea Pearl. Causality: models, reasoning and inference. Cambridge Univ Press, 2000.

Judea Pearl. Principal stratificationa goal or a tool? The International Journal of Biostatistics, 7(1), 2011. 
Donald B Rubin. Causal inference using potential outcomes. Journal of the American Statistical Association, 100(469):322-331, 2005.

Larry Wasserman. Simpson's paradox explained. Blog: Normal Deviate, June 20, 2013. Available at http://normaldeviate.wordpress.com/. 